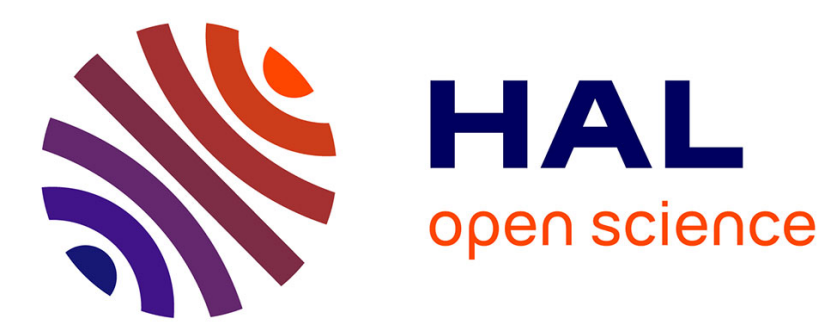

\title{
Temperature MAC Plug-in for Large Scale WSN
}

Walid Bechkit, Yacine Challal, Abdelmalik Bachir, Abdelmadjid Bouabdallah

\section{To cite this version:}

Walid Bechkit, Yacine Challal, Abdelmalik Bachir, Abdelmadjid Bouabdallah. Temperature MAC Plug-in for Large Scale WSN. ICC 2015 - IEEE International Conference on Communications, Jun 2015, London, United Kingdom. hal-01149317

\section{HAL Id: hal-01149317 https://hal.science/hal-01149317}

Submitted on 6 May 2015

HAL is a multi-disciplinary open access archive for the deposit and dissemination of scientific research documents, whether they are published or not. The documents may come from teaching and research institutions in France or abroad, or from public or private research centers.
L'archive ouverte pluridisciplinaire HAL, est destinée au dépôt et à la diffusion de documents scientifiques de niveau recherche, publiés ou non, émanant des établissements d'enseignement et de recherche français ou étrangers, des laboratoires publics ou privés. 


\title{
Temperature MAC Plug-in for Large Scale WSN
}

\author{
Walid Bechkit Member, IEEE, Yacine Challal Member, IEEE, Abdelmalik Bachir, Abdelmadjid \\ Bouabdallah Member, IEEE
}

\begin{abstract}
The quality of radio communication links decreases with high temperatures. In this paper, we investigate the effect of temperature on percolation-based connectivity in large scale wireless sensor networks and show that more energy can be saved by allowing some nodes to go to deep sleep mode when temperature decreases and links improve. We determine a closedform formula for a threshold network density $\lambda^{*}(\tau)=\frac{C_{0}}{\pi R(\tau)^{2}}$ in function of temperature $\tau$. Beyond $\lambda^{*}(\tau)$, the network percolates and guarantees connectivity. Based on this result, we propose a simple yet efficient Temperature-Aware MAC plugin (TAMAC) that enables the underlying MAC protocol to dynamically adapt the network effective density to allow further energy savings while maintaining network connectivity. TA-MAC can be potentially used with any wireless MAC protocol. We carried out simulations and demonstrated that BMAC and SCP-MAC augmented with TA-MAC plugin allow a significant energy efficiency improvement.
\end{abstract}

Index Terms-Wireless Sensor Networks, Temperature Impact, Connectivity, Energy Saving.

\section{INTRODUCTION}

One of the important issues in WSN is network connectivity. Connectivity driven topology management has been widely investigated in the literature and a number of solutions have been proposed. Most of these solutions do not consider the impact of the atmospheric conditions such as temperature on the radio communication range. Temperature has been proven to significantly affect radio communication links negatively, as documented in the data sheets of low power radios [1], [2].

When temperature increases, both transmission power and sensitivity decrease which leads to the deterioration of wireless communication links thereby affecting the overall connectivity of the network [3]. As an example, a network designed to operate under typical conditions, i.e. a temperature of $25^{\circ} \mathrm{C}$, will experience connectivity deterioration when temperature increases above $25^{\circ} \mathrm{C}$. Conversely, when temperature decreases below $25^{\circ} \mathrm{C}$, the radio link quality improves, the network becomes over-dimensioned and some nodes become less necessary for the network to remain connected. These nodes are able to go to deep sleep mode to save energy without affecting the connectivity of the network.

In this paper, we consider percolation-based connectivity and determine a closed-form formula for a threshold network density $\lambda^{*}(\tau)=\frac{C_{0}}{\pi R(\tau)^{2}}$ in function of temperature $\tau$. Beyond $\lambda^{*}(\tau)$, the network percolates and guarantees connectivity. Based on this result, we propose a temperature-aware MAC

Walid Bechkit is with Université de Lyon, INSA-Lyon, CITI-INRIA, Villeurbanne, France. E-mail: walid.bechkit@insa-lyon.fr. Yacine Challal, Abdelmadjid Bouabdallah are with Université de Technologie de Compiègne, HEUDIASYC UMR CNRS 7253, Compiègne, France. E-mails: \{ychallal, bouabdal\}@hds.utc.fr. Abdelmalik Bachir is with the CS Department, University of Biskra, Algeria. E-mail: a.bachir@univ-biskra.dz. plugin that allows the underlying MAC protocol to dynamically adapts the network effective density to allow further energy savings while maintaining network connectivity. TAMAC can be potentially used with any wireless MAC protocol.

This paper is organized as follows: in Section II we present an overview of the research carried out on percolation-based network connectivity. In Section III, we present a mathematical model for wireless links and connectivity conditions. In Section IV, we analyze and model the impact of temperature on the communication range of sensor nodes. Next, we propose in Section $\mathrm{V}$ a temperature-aware MAC plugin that allows the MAC protocol to minimize energy consumption while ensuring network connectivity under varying temperature conditions. In Section VI, we evaluate our solution according to various criteria including the mean power consumption and traffic load. We show that our temperature-aware MAC plugin allows saving significant amount of energy without affecting network connectivity. In section VII, we present our conclusions.

\section{RELATED WORK}

First, it is worthwhile to point out that the connectivity in WSN has been mainly studied from two sides: the percolationbased connectivity and the full connectivity. A fundamental result of the percolation theory is that a geometric graph induced by a Poisson Point Process with a constant density $\lambda$ contains an infinite connected component with non-zero probability if and only if the density is higher than a critical value $\lambda^{*}$, we say then that the percolation occurs. In a network context, a node of this largest component can communicate with a high portion of the network. However, we may have many small disconnected components. The existence of an infinite connected component was considered sufficient in many research works, we refer to this paradigm as percolationbased connectivity. The full connectivity (resp. $k$-connectivity) on its turn guarantees that each pair of nodes can communicate with each others using at least one path (resp. $k$ paths). The conditions of such connectivity were widely studied in the literature and a lot of relations were derived. As we consider large scale WSN, we will focus in what follows on solutions relying on percolation based connectivity.

In [4], Godfrey and Ratajczak proposed Naps, a completely distributed topology control protocol for ad-hoc and sensor networks. Authors considered a network deployed according to an infinite Poisson Point Process (PPP) with a high density, they rely on the percolation-based connectivity and assumed the existence of a critical threshold $\lambda^{*}$ such that for all density $\lambda>\lambda^{*}$, there is a non-zero probability that a particular node is in an infinite connected component. In Naps, each 
node waits for a random small amount of time and then starts its time period of length $\mathrm{T}$. At the beginning of this period, it broadcasts a hello message to all its neighbors. If it receives more than $c$ reply messages, it switches off its radio and go to sleep until the end of the period. Otherwise, it remains active for the whole period. The parameter $c$ represents then a threshold value of the average node degree allowing the network connectivity based on percolation. Its value is computed depending on the target density $\lambda^{*}$.

In [5], authors propose a solution very similar to Naps [4] while relying also on the percolation-based connectivity. Authors propose that each node goes to sleep with a probability $\frac{\phi}{\langle N\rangle}$ at each round where $\langle N\rangle$ is the degree of the node. Using a degree-dependent dynamic site percolation process modeling, authors proved the existence of the constant $\phi(2<\phi<\infty)$ such that the percolation occurs.

On another side, the effect of temperature has been validated independently by many researchers in the literature (e.g. [6] [7]). In [8] and [7], the authors studied through experiments the impact of the temperature on wireless communication links and data delivery performance in WSN outdoor deployments for industrial process. In [6], the authors separated the effect on the transmitter from the one on the receiver. However, the model they established only provides the reduction in link quality in function of the combined effects at both the transmitter and the receiver. A more accurate information about the effect of temperature on the radio transceivers can be directly obtained from radio circuits data sheets. The effect of temperature is also studied in [9].

As far as we can say, temperature impact on radio link quality has not been taken into consideration in designing MAC protocols for energy saving in low power networks while maintaining network connectivity. This is the aim of our solution that we present through the following sections.

\section{SYSTEM MODEL}

\section{A. Wireless Network Model}

We consider a wireless network where nodes are distributed according to a Poisson Point Process of constant spatial intensity $\lambda$ in a surface $\mathcal{A}$. All nodes transmit with a full power $P_{t}$. The power of the signal emitted by Node $i$ and received by Node $j$ is $P_{t} L\left(d_{i j}\right)$, where $L($.$) is the attenuation function$ in the wireless medium.

The uncoordinated nature of communication allows multiple transmitters to communicate at the same time, however, creates interference at all receivers. A common connection model in ad-hoc networks is the signal-to-interference noise ratio (SINR) model, where two nodes are connected if the SINR between them is greater than a threshold.

In other words node $i$ can transmit data to node $j$ if the signal received by $j$ is strong enough, compared to noise. Formally, this condition is written as

$$
S I N R_{i j}=\frac{P_{t} L\left(d_{i j}\right)}{N_{0}+\gamma \sum_{k \neq i, j} P_{t} L\left(d_{k j}\right)} \geq \beta
$$

where $N_{0}$ is the power of the thermal background noise and $\beta$ is the signal to noise ratio required for successful decoding.
The coefficient $\gamma$ weights the effect of interferences, depending on the orthogonality between codes used during simultaneous transmissions. Without loss of generality, we will consider $L\left(d_{i j}\right)=a d_{i j}^{-b}$, where $a$ and $b$ are parameters that describe the wireless channel, $b$ is usually called path loss exponent.

Using this transmission condition, the network can be modeled with a graph $G(N, E)$ where $N$ is the set of nodes, and $E=(i, j) \in N \times N$, st. $S I N R_{i j} \geq \beta$.

\section{B. Radio Communication Range}

We define the radio communication range $R$ as the range that allows a successful transmission according to the condition in equation 1 , in the absence of interference $(\gamma=0)$. In this case, if we neglect the thermal floor noise $(\gamma=0$ and $N_{0}=0$ ) the successful transmission depends on the power of the received signal, referred to as $P_{r}$, which is equal to the power radiated by transmitter after attenuation:

$$
\begin{aligned}
P_{r} & =P_{t} L(d) \\
& =P_{t} a d^{-b}
\end{aligned}
$$

The minimum power of $P_{r}$ that allows successful transmission is called sensitivity, and is referred to as $P_{s}$.

The previous equation (3) can be used to express the relation between the sensitivity $P_{s}$ and the communications range $R$. We have:

$$
P_{s}=a R^{-b} P_{t}
$$

Thus, the radio communication range can be quantified according to the following equation:

$$
R=\left(a \frac{P_{t}}{P_{s}}\right)^{1 / b}
$$

If we consider that our sensor network is deployed out-door, one of the most accurate model for the path loss is the two-ray ground propagation model. In the two ray ground model, the values of $a$ and $b$ are the following:

$$
\begin{aligned}
a & =G_{t} G_{r} H_{t}^{2} H_{r}^{2} \\
b & =4
\end{aligned}
$$

where $G_{t}$ (resp. $G_{r}$ ) is the antenna gain at the transmitter (resp. receiver), and $H_{t}$ (resp. $H_{r}$ ) is the antenna height at the transmitter (resp. receiver).

\section{Percolation condition under the physical model}

Kleinrock and Silverster [10] studied the conditions to have an asymptotically connected network in radio networks. Authors assume that nodes are randomly distributed according to a Poisson Point Process (PPP) and found that the minimum required number of neighbors is equal to six, the 'magic number' as they called it. This 'magic number' was after that revised to eight in [11].

In [12] authors, proved that there is no magic number and that if the number of neighbors is constant the network will be disconnected when the region became large. They showed that for an infinite area there exists an infinite connected component (percolation) with non zero probability if the 
number of neighbors $\pi R^{2} \lambda>C_{0}$ for some critical value $2.198<C_{0}<10.526$.

Following this condition, the minimum density required to induce network percolation is then given by :

$$
\lambda^{*}=\frac{C_{0}}{\pi R^{2}}
$$

The minimal density in equation 8 that induces network percolation has been derived for networks without interference $(\gamma=0)$. Dousse et al. have demonstrated in [13] that if a threshold density $\lambda^{*}$ exists, with $\gamma=0$ (no interference), so that $\forall \lambda \geq \lambda^{*}$ the network percolates, then under the generalized physical model with interferences, there exists $\gamma>0$ such that the network percolates $\forall \lambda \geq \lambda^{*}$. In other words, if some threshold density $\lambda^{*}$ induces network percolation under the simplified model ( $\gamma=0$, no interferences) then there exist some orthogonal coding through time or frequencies $(\gamma>0)$ such that the network percolation occurs also.

Following this result, we will consider $\lambda^{*}$ in equation 8 as the critical value that induces network percolation under the generalized physical model.

\section{EFFECT OF TEMPERATURE}

\section{A. On Communication Range}

There is increasing evidence that high temperature affects both the transmitter and the receiver. In [8] and [7], Boano et al. studied through experiments the impact of the temperature on wireless communication links and data delivery performance in WSN outdoor deployments for industrial process. In [6], the authors separated the effect on the transmitter from the one on the receiver. However, the model they established only provides the reduction in link quality in function of the combined effects at both the transmitter and the receiver. A more accurate information about the effect of temperature on the radio transceivers can be directly obtained from radio circuits data sheets. For instance, the data sheet of the widely used low-power radio CC2400 [1] provides quantitative information which shows that the values of $P_{s}$ and $P_{t}$ are not constants but are affected by temperature change. Therefore, we use the the notation $P_{t}\left(\tau_{t}\right)$ (resp. $P_{s}\left(\tau_{r}\right)$ ) to express the variation of the transmission power (resp. sensitivity) in function of the temperature $\tau_{t}$ (resp. $\tau_{r}$ measured at the transmitter (resp. receiver). Consequently, the radio communication range is also affected by the temperature at the transmitter and the receiver. Therefore, we have:

$$
R\left(\tau_{t}, \tau_{r}\right)=\left(a \frac{P_{t}\left(\tau_{t}\right)}{P_{s}\left(\tau_{r}\right)}\right)^{1 / b}
$$

The forms of the function $P_{t}(\tau)$ (resp. $P_{s}(\tau)$ ) depend on the radio chips. Some manufacturers provide data about this variation for some temperature values, e.g. CC2400 [1] and CC2530 [2]. A closed-form expression for $P_{t}\left(\tau_{t}\right)$ (resp. $\left.P_{s}\left(\tau_{r}\right)\right)$ is not provided. However, the data provided in the data sheet allows to use regression to find the closed polynomial representations for $P_{t}\left(\tau_{t}\right)$ and $P_{s}\left(\tau_{r}\right)$.

\section{B. On Percolation}

As shown in section IV-A, the transmission range is not fixed but changes according to temperature at the transmitter $\tau_{t}$ and temperature at the receiver $\tau_{s}$. For the sake of simplicity, we consider that the network is deployed over homogeneous area in which the same temperature is measured at all the nodes, i.e. $\tau_{t}=\tau_{r}$. In this case, we use $\tau$ to refer to temperature. For simplicity, we use the notation $R(\tau)$ instead of $R\left(\tau_{t}, \tau_{r}\right)$. Similarly, the critical density that induces network percolation also depends on the temperature. Therefore, we have:

$$
\lambda^{*}(\tau)=\frac{C_{0}}{\pi R(\tau)^{2}}
$$

\section{TEMPERATURE-AWARE MAC PLUGIN FOR ENERGY SAVING}

In this section, we propose a simple yet efficient MAC plugin that adapts node's transceiver activity to ensure network connectivity while minimizing energy consumption, under varying temperature conditions. The underlying algorithm switches on and off nodes' transceivers in function of temperature. This algorithm guarantees that only those nodes that need to be on to ensure percolation and/or send data are operating. The others switch their transceivers off to save energy.

We use in what follows $\tau^{+}$to refer to the maximum temperature. To ensure connectivity in the worst case, the initial density of nodes should be equal to $\lambda_{\tau^{+}}^{*}$. We assume that $\mathcal{A} \lambda_{\tau^{+}}^{*}$ nodes are initially deployed over the deployment area $\mathcal{A}$ following a Poisson Point Process. We assume also that nodes use the default transmission power level which is supposed to be the maximum transmission level (This is the case of most radio chips like CC2400, CC2420, CC1100, etc.). When the temperature $\tau$ is lower than $\tau^{+}$, a lower density is sufficient. We propose thus to put some nodes in sleep mode, and by doing so a sizable amount of energy can be saved.

We propose to calculate $\rho_{\tau}=\lambda_{\tau}^{*} / \lambda_{\tau^{+}}^{*}$ which represents the ratio of network density that is required to maintain connectivity when the temperature is $\tau$ compared to the initial deployment where the density is calculated according to the highest temperature. The value of $\rho_{\tau}$ also represents the number of nodes required to be on when temperature is equal to $\tau$ compared to that when temperature is equal to $\tau^{+}$.

By using equation (10), we obtain:

$$
\begin{aligned}
\rho_{\tau} & =\frac{\lambda(\tau)}{\lambda\left(\tau^{+}\right)} \\
& =\frac{C_{0}}{\pi R(\tau)^{2}} \times \frac{\pi R\left(\tau^{+}\right)^{2}}{C_{0}} \\
& =\frac{R\left(\tau^{+}\right)^{2}}{R(\tau)^{2}}
\end{aligned}
$$

The idea of our approach is then to keep the ratio of active nodes equal to $\rho_{\tau}$ at temperature $\tau$. Note that, by selecting nodes which go off randomly, the distribution of the nodes which remain on maintains the same characteristics, i.e. distributed according to PPP (thanks to the thinning property of PPP). We also assume that each node is able to know its temperature (temperature sensor readings, extraction from log 
files, computation, expectation, etc.). Our approach called TAMAC relies on algorithm 1 that is executed by every node periodically (a predetermined period of time) without the need to exchange information with other nodes.

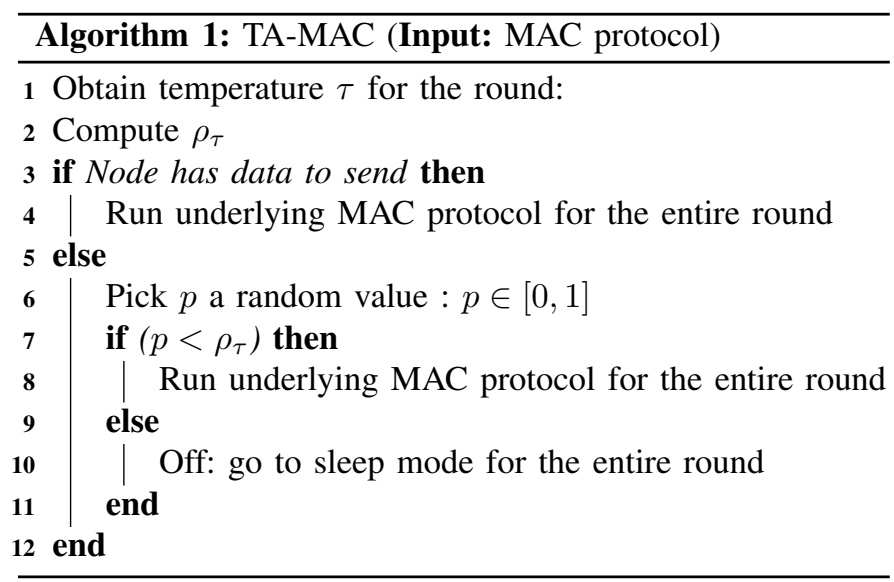

\section{PERformance Evaluation}

In this section, we evaluate the energy efficiency of the proposed solution and compare it to existing MAC protocols that are not temperature-aware. First, we present the temperature model and the MAC protocols along with the energy model used in simulations. Next, we give and discuss the simulation results.

\section{A. Temperature model}

We refer to the ERBS's model [14] as derived in [15] where the average normalized diurnal temperature variation is represented by:

$$
\begin{aligned}
\tau(y, m, t)= & \tau(y, m)+A_{\tau}(y, m)[0.4632 \cos (\gamma(t)-3.805) \\
& +0.0984 \cos (2 \gamma(t)-0.360) \\
& +0.0168 \cos (3 \gamma(t)-0.822) \\
& +0.0138 \cos (4 \gamma(t)-3.513)]
\end{aligned}
$$

where $\gamma(t)=2 \pi(t-1) / 24 ; \mathrm{t}$ is the time in hour; $\tau(y, m)$ is the daily monthly mean air temperature; and $A_{\tau}(y, m)$ is the monthly mean thermal amplitude in degrees Celsius [15]. For $\tau(y, m)=A_{\tau}(y, m)=25^{\circ} \mathrm{C}$, figure 2 top shows the temperature variation throughout the day (time in hours).

\section{B. MAC protocols}

In order to evaluate the efficiency of TA-MAC, we will consider two MAC protocols for WSN: BMAC [16] and SCPMAC [17]. BMAC is one of the most deployed protocols in WSN as it is the default protocol in most of the sensor node prototypes (Micaz, Mica-2, etc.) that rely on the operating system TinyOs. BMAC achieves high energy savings through the use of preamble sampling technique (see [18] for a survey on these protocols) where nodes spend most of their time in sleep mode, but only wake up from time to time to check for incoming traffic. To make sure that a node does not miss an incoming data packet, each node prepends its data packet transmission with a preamble that is long enough so that any potential receiver will wake up during the transmission of the preamble. When a node wakes up and detects a preamble being transmitted, it keeps receiving it until it decodes the data packet. A more advanced version of preamble sampling is SCP-MAC in which nodes synchronize on a common sleep/wake schedule to reduce the overhead of sending long preambles.

\section{Energy consumption model}

We propose to evaluate $\mathcal{P}$ (Joules/s) the average power consumed by a node. The power $\mathcal{P}$ depends on many parameters such as the energy consumption of the micro-controller and the radio. For the sake of conciseness and simplicity, we consider only the power consumed by the radio which is dominant. The energy consumed by the radio depends on the underlying MAC protocol.

We use the analytical model developed in [17] for the evaluation of the energy consumption of the BMAC [16] and SCP-MAC [17] protocols. We have:

$$
\mathcal{P}=\mathcal{P}_{t x}+\mathcal{P}_{r x}+\mathcal{P}_{p l}
$$

where $\mathcal{P}_{t x}$ (resp. $\mathcal{P}_{r x}$, and $\mathcal{P}_{p l}$ ) is the average power drained in transmission (resp. reception and channel sampling). We evaluate the mean power consumption on the flooding; therefore, each node transmits one packet and receives $\eta(\tau)$ packets, where $\eta(\tau)$ is the number of neighbors. The average power drained during transmission (resp. reception and channel sampling) is:

$$
\begin{aligned}
\mathcal{P}_{t x} & =\left(T_{p}+T_{\text {data }}\right) \cdot P_{t x} \cdot r_{\text {data }} \\
\mathcal{P}_{r x} & =\eta(\tau) \cdot\left(T_{p} / 2+T_{\text {data }}\right) \cdot P_{r x} \cdot r_{\text {data }} \\
\mathcal{P}_{p l} & =\left(T_{p l} / T_{p}\right) \cdot P_{p l}
\end{aligned}
$$

where $P_{t x}$ (resp. $P_{r x}$, and $\left.P_{p l}\right)$ is the power drained during the transmission (resp. reception and channel sampling). $T_{\text {data }}$ is the transmission duration of one data packet, $T_{p}$ is the channel sampling interval, $T_{p l}$ is the duration of channel sampling, and $r_{\text {data }}$ is the packet rate. We note that we assume that there are no transmission errors, and thus no retransmissions. We note that $P_{p l}$ which is the power drained during the sampling of the channel can be considered to be equal to $P_{\mathrm{rx}}$.

\section{Simulation results}

We conducted extensive simulations in order to evaluate the power consumption of our approach and compare it to MAC protocols that are not temperature-aware. We evaluate also the impact of our solution on the network connectivity. We give in Table I the common simulation parameters.

1) Impact of temperature on power consumption: In this subsection, we compare power consumption when varying temperature from $-20 C$ to $+80 C$. We plot in Figure 1 the impact of temperature on power consumption per node when 
TABLE I

SUMMARIZE OF THE SIMULATION PARAMETERS

\begin{tabular}{|c|c|c|}
\hline \multirow{3}{*}{ CC2400 radio } & $V$ & $3 \mathrm{v}$ \\
& $I_{r x}$ & $24 \mathrm{~mA}$ \\
& $I_{t x}$ & $19 \mathrm{~mA}$ \\
& data rate & $250 \mathrm{kbps}$ \\
\hline \multirow{3}{*}{ Radio propagation (TRG) } & $H_{r}$ & $1.5 \mathrm{~m}$ \\
& $H_{t}$ & $1.5 \mathrm{~m}$ \\
& $G_{r}$ & 1 \\
\hline \multirow{2}{*}{ MAC Layer } & $G_{t}$ & 1 \\
\hline & $r_{d a t a}$ & $(1 / 300)$ Packet/s \\
& $T_{\text {data }}$ & 50 Bytes $\times 32 \cdot 10^{-6}$ s/Byte \\
& $T_{p}$ & $200 \cdot 10^{-3} \mathrm{~s}$ \\
& $T_{p l}$ & $2.5 \cdot 10^{-3} \mathrm{~s}$ \\
\hline
\end{tabular}

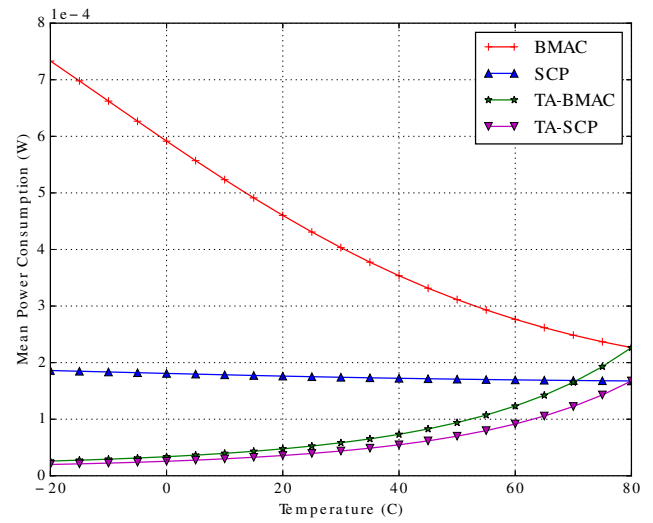

Fig. 1. Impact of Temperature on Power Consumption

using BMAC and SCP-MAC as MAC protocols and when using the same protocols augmented with TA-MAC algorithm.

The figure shows that TA-MAC approach presents a significant improvement in terms of power consumption compared to MAC protocols without temperature awareness. This is due to the proposed temperature-aware sleep scheduling. Indeed, at each round a fraction of nodes $\left(1-\rho_{\tau}\right)$ turn their radio off which allows to save a significant amount of energy.

2) Average power consumption: In this subsection, we compare the average power consumption per node when using BMAC and SCP-MAC as MAC protocols and when using the same protocols augmented with our Temperature-Aware MAC approach. We recall that nodes are initially deployed considering the highest possible temperature (with a density equal to $\lambda_{\tau^{+}}$).

The mean power consumption when a given node is on is given in Eq. (14) and we assume that the power consumption when the node is off is equal to zero. Indeed, as explained before, the most important amount of energy is consumed by the radio and the power consumption can be negligible whenever nodes turn their radios off. We plot in Figure 2 the obtained results when considering the CC2400 radio chips and the two ray ground model (parameters are given in Table I).

Figure 2 shows that by using the basic MAC protocols where nodes do not use temperature aware energy saving,

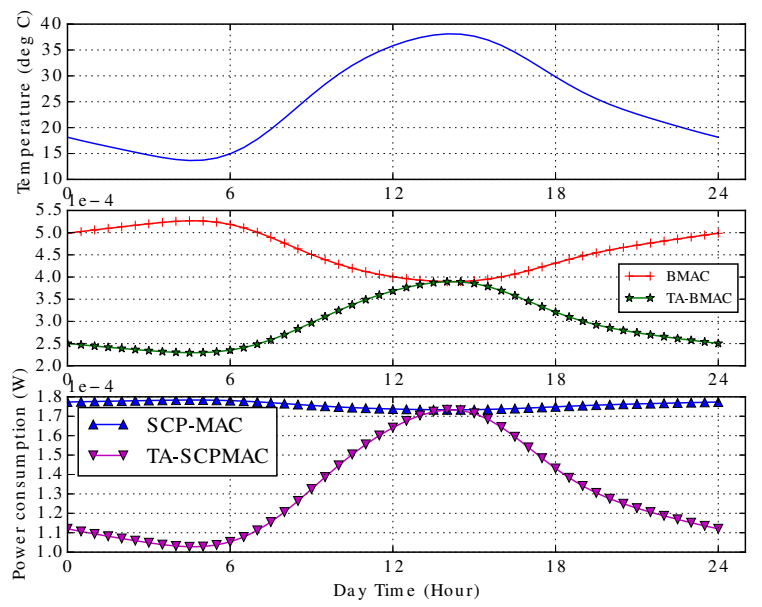

Fig. 2. Top: Temperature variations. Bottom: Mean power consumption per node

the average power consumption increases when the temperature decreases and vice versa. Indeed, when the temperature decreases the communication range becomes higher and the number of potential neighbors of each node increases which increases the power drained by the data reception.

On the other hand, the figure shows clearly that the TAMAC algorithm outperforms the other approaches thanks to sleep scheduling. When the temperature decreases, the communication range becomes higher which reduces the fraction of nodes required to guarantee the network percolation. This allows to save more energy since more nodes will turn off their radios. The results show that the TA-MAC algorithm allows to save a sizeable amount of energy which ranges from $0 \%$ when the temperature is maximum to about $50 \%$ (for BMAC) and $40 \%$ (for SCP-MAC) when the temperature is minimum.

3) Impact of data rate on energy savings: In Figure 3, we study the effect of traffic load variations on the mean power consumption of our Temperature MAC Plugin. We show that there is a significant difference between BMAC and SCP-MAC without our Temperature MAC Plugin and the same protocols with the Plugin. The difference in energy consumption is particularly high with BMAC because nodes running BMAC use long preambles and thus consume more energy than those running SCP-MAC in which nodes spend more time in sleep mode. Therefore, the effect of using Temperature MAC Plugin is less visible with MAC protocols having a very high sleep mode ratio and in lighter traffic loads.

4) Impact of Check Interval variation: In Figure 4, we vary the check interval $\left(T_{p}\right)$ and plot the induced mean power consumption. For BMAC, we notice that there is an optimal value $(0.075 \mathrm{~s}$ for the configuration setup we used) for the check interval where the mean power consumption is minimized (Note that BMAC has a limited duty cycle [16] because longer check intervals involve longer preamble transmissions and result in higher energy consumption. Therefore, the amount of energy savings that can be achieved with BMAC is limited). For values smaller than $0.075 \mathrm{~s}$ more energy is 


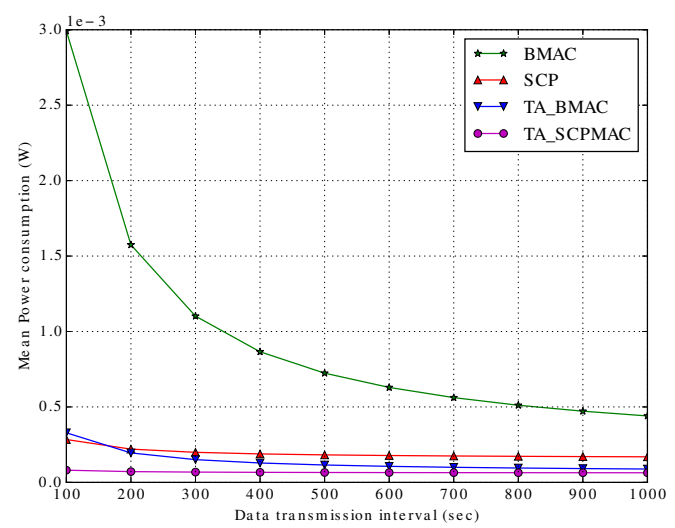

Fig. 3. Energy savings with respect to data rate

consumed because nodes spend more time in checking the channel waiting for incoming data. Smaller check intervals are only interesting for delay constrained applications. However, we notice that the optimal value for the check interval with TA-BMAC is significantly larger than that of BMAC which offers the possibility of using higher duty cycles thereby allowing higher amounts of energy savings. The reason why TA-BMAC has a higher check interval is because it has smaller number of neighbors which limits the amount of energy spent at the reception side. Also, with TA-BMAC, nodes that are not selected to continue to operate normally, will go into deep sleep mode where they do not transmit packets, which results in lesser energy spent in transmission (particularly the long preamble). For SCP, there is no limitation for the check interval and the energy consumption continues to decrease when the check interval increases. The same behavior is observed with TA-SCP.

\section{CONCLUSION}

In this paper, we proposed a temperature-aware MAC plugin that can be used with potentially all existing WSN MAC protocols to make them even more energy efficient in environment where temperature variations are frequent and significant. We have shown that TA-MAC is relatively simple to implement and provides substantial benefits for large scale networks by adapting node's transceiver activity to ensure percolationbased connectivity under varying temperature conditions.

\section{ACKNOWLEDGMENT}

This work is funded in part by the French Government, through the program Investments for the future managed by the National Agency for Research (Reference ANR-11-IDEX0004-02), and under the framework of the Labex MS2T, the Algerian Ministry of Higher Education and Scientific Research under contract $\mathrm{B} * 01420130132$.

\section{REFERENCES}

[1] Chipcon Corporation, "CC2400 2.4 GHz Low-Power RF Transceiver, Data Sheet," 2008.

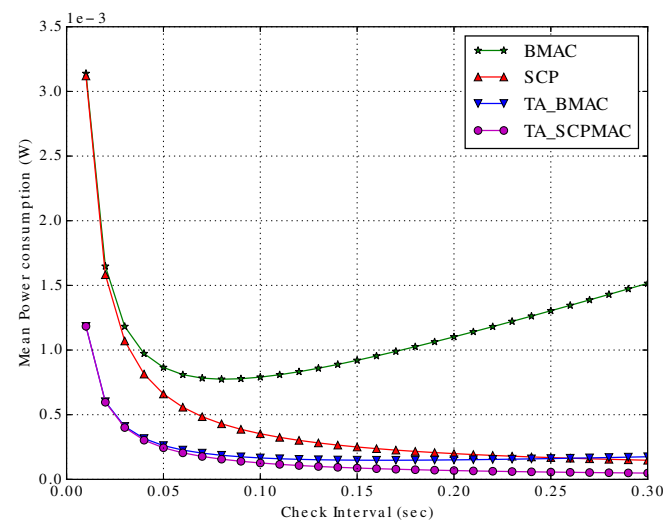

Fig. 4. Energy savings in function of check interval

[2] Texas Instruments, "CC2530 A True System-on-Chip Solution for 2.4GHz IEEE 802.15.4 and ZigBee Applications, Data Sheet," 2009.

[3] A. Bachir, W. Bechkit, Y. Challal, and A. Bouabdallah, "Temperatureaware density optimization for low power wireless sensor networks," IEEE Communications Letters, vol. 17, no. 2, pp. 325-328, 2013.

[4] P. Godfrey and D. Ratajczak, "Naps: scalable, robust topology management in wireless ad hoc networks," in IPSN 2004, pp. $443-451$.

[5] Z. Kong and E. M. Yeh, "Distributed energy management algorithm for large-scale wireless sensor networks," in ACM MobiHoc 2007, (New York, NY, USA), pp. 209-218.

[6] K. Bannister, G. Giorgetti, and S. Gupta, "Wireless sensor networking for hot applications: Effects of temperature on signal strength, data collection and localization," in Proceedings of the 5nd IEEE workshop on Embedded Networked Sensors, (Charlotteville, VA, USA), 2008.

[7] C. A. Boano, J. Brown, N. Tsiftes, U. Roedig, and T. Voigt, "The impact of temperature on outdoor industrial wsn applications," IEEE Trans. Industrial Informatics, vol. 6, no. 3, pp. 451-459, 2010.

[8] C. A. Boano, J. Brown, Z. He, U. Roedig, and T. Voigt, "Low-power radio communication in industrial outdoor deployments: The impact of weather conditions and atex-compliance," in SENSAPPEAL, pp. 159$176,2009$.

[9] N. Baccour, A. Koubaa, H. Y. Marco Zuniga, C. A. Boano, and M. Alves, "Radio link quality estimation in wireless sensor networks: a survey," ACM Trans. Sen. Netw., vol. 8, November 2012.

[10] L. Kleinrock and J. Silvester, "Optimum transmission radii for packet radio networks or why six is a magic number," in National Telecommunications Conference (NTC '78), vol. 1, pp. 431-435, 1978.

[11] H. Takagi and L. Kleinrock, "Optimal transmission ranges for randomly distributed packet radio terminals," IEEE Transactions on Communications, vol. COM-32, pp. 246-257, March 1984.

[12] T. K. Philips, S. S. Panwar, and A. N. Tantawi, "Connectivity properties of a packet radio network model," Information Theory, IEEE Transactions on, vol. 35, no. 5, pp. 1044-1047, 1989.

[13] O. Dousse, M. Franceschetti, N. Macris, R. Meester, and P. P. Thiran, "Percolation in the signal to interference ratio graph," Journal of Applied Probability, vol. 43, no. 2, pp. 552-562, 2006.

[14] D. G. Erbs, Models and applications for weather statistics related to building heating and cooling loads. $\mathrm{PhD}$ thesis, Mechanical Engineering Dept., University of Wisconsin Madison, 1984.

[15] J. Bilbao, A. H. D. Miguel, and H. D. Kambezidis, "Air temperature model evaluation in the north mediterranean belt area," J. of applied meteorology, vol. 41, pp. 872-884, 2002.

[16] J. Polastre, J. Hill, and D. Culler, "Versatile low power media access for wireless sensor networks," in SenSys '04, (New York, NY, USA), pp. 95-107, ACM, 2004.

[17] W. Ye, F. Silva, and J. Heidemann, "Ultra-low duty cycle mac with scheduled channel polling," in Proceedings of ACM SenSys, pp. 321334, 2006.

[18] A. Bachir, M. Dohler, T. Watteyne, and K. K. Leung, "Mac essentials for wireless sensor networks," Commun. Surveys Tuts., vol. 12, pp. 222248, Apr. 2010. 